\title{
The Role of Sport Science in the New Age of Digital Sport
}

As the editors of the International Journal of Sports Physiology and Performance, we observe that more technology-driven studies are being submitted to our journal. Some of these studies are developments of new technological solutions and therefore not within our scope, while others are validating or using technology to gain valuable insights into sport physiology and performance.

Technology-driven digital solutions may provide knowledge beyond what standard measurements have previously allowed. Positioning systems, inertial movement units, and various sensors that measure physiological responses have become both small and accurate enough to provide valid information from athletes' training and competitions. Many of these digital tools are already used as part of sport practice for various purposes, such as evaluating individual or collective behavior during training or competitions, developing sportspecific benchmarks, and providing a framework for load management, training prescription, and recovery strategies. Although some of these solutions provide helpful scientifically backed decision support, others give nonvalidated information that can either confuse or lead to wrong decision making among practitioners.

The main future possibilities in the new age of digital sport include the following aspects:

- Measuring physiological and performance parameters during relevant sport-specific situations, thereby gathering comprehensive and complementary data from the daily training and testing process. This work has high relevance for long-term development of athletes. While most previous measurements have been done episodically in the laboratory, smaller and more accurate sensors can now continuously and seamlessly be used in real-life situations, including competitions.

- Advanced mathematical models that enable intelligent analyses of information from various sources open up possibilities to interpret data beyond standard statistical methods and with more precision than our human capability to observe associations in complex data.

- Development of digital twins (ie, models that can represent the physiological state of an individual) provides individualized decision support, communicated by "digital coaches" that are designed to motivate to change behavior and thereby improve performance.
However, these possibilities require further development, and there are related challenges as follows:

- There is a risk of taking cutoffs with too much focus on the possibilities given by technology and data-driven results. We should meet this challenge by solving the same problems with different approaches, being open minded toward data-driven results, testing hypotheses derived from these approaches, and strive to understand the mechanisms underlying the new findings.

- We should also acknowledge that a good coach is able to observe nuances that measurements cannot detect. Information provided by technology can lack the holistic context needed for taking optimal decisions among athletes and coaches. In this connection, many groups are also measuring more variables than they can digest and/or understand.

- There is also a risk of losing human interaction by uncritically trusting technology. We have to ensure that digital technology does not replace the human dialogue but facilitates evidencebased discussions between scientists and coaches, as well as between athletes, coaches, and their support teams.

An important consequence for successfully meeting the future of digital sport is to develop good service systems, where valid technological solutions are part of the support system, and where athletes and coaches are well educated on how to use technology optimally as part of their development process. Therefore, sport scientists should critically evaluate digital solutions, their decision support, and the effect they have on improving performance. Here, domain competence is crucial for successfully developing useful products or services, and scientists in the field of sport physiology and performance should play an important role in the multidisciplinary work required for technologically driven projects in sports.

The same issues apply for comparable services in other parts of society, such as the health care and medical sectors. If sport scientists are at the frontiers of handling these issues, we will not only help to digitally transform sport but also be a role model for approaching the new age of digital society.

Øyvind Sandbakk, Associate Editor, IJSPP 